\title{
Building youth and family resilience for better mental health: developing and testing a hybrid model of intervention in low- and middle-income countries
}

Debasish Basu, Sugandha Nagpal, Renjith R. Pillai, Victoria Mutiso, David Ndetei and Kamaldeep Bhui

\section{Summary}

Resilience is a dynamic, multi-level, multi-systemic process of positive adaptation at the individual, family and community levels. Promoting resilience can be a cost-effective form of preventive and early intervention, offering significant health advantages for young people throughout their lives. Developing resiliency interventions for youth and their families in low- and middle-income countries (LMICS), particularly in the context of the ongoing pandemic, is especially important given a lack of services and trained specialists, and poor levels of public spend on mental health, alongside marked and clustered psychosocial disadvantages and adverse childhood experiences. We propose a 'hybrid' model targeting 10- to 17 year-old children and their families, and options to engage through communities, schools and the family unit. These options will enhance individual and family resilience, and possibly buffer against adversity. The adaptations respect cultural and health beliefs, take account of structural drivers of inequalities and are suitable for LMICs.

\section{Keywords}

Resilience; low- and middle-income countries; youth; family; integrated intervention.

\section{Copyright and usage}

(c) The Author(s), 2021. Published by Cambridge University Press on behalf of the Royal College of Psychiatrists.
Debasish Basu is Head of the Department of Psychiatry at the Postgraduate Institute of Medical Education and Research in Chandigarh, India. His interests include addiction psychiatry and social psychiatry. Sugandha Nagpal is an Assistant Professor of Sociology at Jindal School of International Affairs at O.P. Jindal Global University, India. Her interests include migration, education and gender. Renjith R. Pillai is an Assistant Professor of Psychiatric Social Work with the Department of Psychiatry at the Postgraduate Institute of Medical Education and Research in Chandigarh, India. He is also a consultant faculty member of the Drug Deaddiction Treatment Centre in Chandigarh India, specialising in child and adolescent psychiatry. Victoria Mutiso holds PhD and MSc degrees in clinical psychology from the University of Nairobi, Kenya. She leads research at the African Mental Health Research and Training Foundation in Kenya. David M. Ndetei is a Professor of Psychiatry at the University of Nairobi, Kenya. He is also the Director of the Africa Mental Health Research and Training Foundation and the WPA Collaborating Centre for Research and Training at the University of Nairobi, Kenya. Kamaldeep Bhui is a Professor of Psychiatry at the University of Oxford, UK, and Director of the UK WPA Collaborating Centre and the Synergi Collaborative Centre on ethnic inequalities in mental illness.

Promoting resilience in general, and psychological resilience specifically, has gained tremendous traction, especially in the context of the COVID-19 pandemic. What explains this interest? We know adversities increase the risk of mental health problems, but not everyone exposed to adversity manifests poor mental health, and many do not develop an illness or recover fully and quickly. Some even show post-traumatic growth toward greater functional levels. Thus, the term resilience is increasingly relevant as a potential focus of prevention and intervention, questioning undue emphasis on deficits and embracing strength-based approaches. Importantly, preventive psychiatry approaches are relevant for agencies seeking to support people living with mental illnesses and for public health efforts for prevention, health promotion and harm reductions.

\section{The shifting concepts of resilience}

From the initial inquiries into 'stress resistance' or 'invulnerability to stress' that assumed resilience to be a stable 'trait' of an individual, the emphasis gradually shifted toward resilience as a dynamic process, demonstrating marked flexibility of responses to adversity rather than an immutable characteristic of a person. With this came the realisation - and the hope - that resilience can be fostered or enhanced, reflecting modifiable social, emotional and behavioural factors. Resilience is now recognised to be a function of interactions between the individual (biological and psychological factors), family, school, workplace, community and organisations, acting within a system of relationships and networks. All of these are determined partly by geopolitical influences that lead to stable safe societies or conflict, poverty and adversity. This necessarily complicates the development of resiliency interventions, as without a systemic focus, interventions at one level are likely to be ineffective if the surrounding levels constrain, negate or oppose positive change. Even individual biological and psychological resilience operate as interdependent components of complex systems that confer adaptation and stress resilience. Cultural beliefs and sociocultural contexts also influence risk definitions and perceptions and meaning-making, classifications and articulation of resiliency factors, and what counts as a meaningful outcome.

\section{A hybrid approach: the role of individual and family factors}

A recent systematic review of resilience-enhancing factors critiques 22 studies and found supportive evidence for 1 community, 6 family and 13 individual factors. ${ }^{1}$ The individual factors were in the domains of cognitive, emotion regulation, social interaction/attachment and personality/self-concept. Many of these individual-level factors can be addressed by the school-based Life Skills Education (LSE) modules advocated by the World Health Organization, and with a reasonably large body of evidence. However, the supportive family resilience factors evidenced by Fritz et $\mathrm{al}^{1}$ are not addressed by the LSE. These family factors are: high positive parenting, high family cohesion, high extended family support, high immediate 
family support, high parental involvement and positive family climate. The single community-level resilience factor with evidence was high social support.

The pivotal role of the family is often neglected in youth resiliency programmes. It is the family that protects, shelters and nurtures the individual in the context of adversity affecting multiple domains of function (e.g. domestic, academic and occupational, among others) and, most importantly, provides an essential link between the individual and the larger community. ${ }^{2}$ Although relevant globally, this is especially true for non-Western and low- and middle-income countries (LMICs), where lifestyles and values are still very family-centric, and societies are more collectivistic than individualistic. In this context, it may be of immense value to promote resilience through a hybrid family-individual approach. Although studies have documented resilience enhancement through either individual or family-based approaches from LMICs, ${ }^{3}$ there is little evidence, as yet, of developing and testing such a hybrid approach in LMIC settings. ${ }^{4}$ Our hybrid approach aims to combine the best elements of the individual LSE-based approach with the family systems-based approach in an integrated intervention setting, and hence hopes to improve upon the existing approaches.

\section{A few of the challenges}

Along with the challenges set out above in designing and testing an intervention to enhance resilience, there are two additional challenges: both the content and the delivery of the intervention need careful design, deliberation and testing for diverse identities and place contexts, geo-political influences and health economies. The content and formats of the intervention will likely vary, not only from the Westernised cultural contexts where most of the published evidence comes from, but also among different LMICs with their own cultures and contexts. Thus, simply 'copy-pasting' an intervention - either at the individual or family level - may not work, and different cultures have to work out their own intervention packages according to the local context of challenges, resources and desirable outcomes. On the other hand, the interventions have to be practical, and have a basic unifying theoretical and heuristic thread irrespective of local adaptation and implementation. This culture-specific versus culture-free consideration needs to be weighed. The second consideration is the delivery mode. Although many earlier studies, especially those in LMICs, used in-person modes of delivery (in individual or group formats), this does not work as well for isolated rural communities, or communities with poor levels of literacy and basic provisions such as shelter and housing, food and water. The COVID-19 pandemic has raised even more challenges as interventions in large gatherings at school, families or communities are no longer viable in the short and perhaps longer term. However, the pandemic has also forced a rapid re-think on the role of remote delivery through telephone, internet and electronic/digital applications. This still may exclude some groups with specialist needs, given the relatively lower accessibility to these technological advances and lower digital literacy and/or aptitude among many sections of LMIC societies.

With these considerations in mind, we have evolved a new integrated intervention, making use of the family resilience model ${ }^{2}$ and the World Health Organization's LSE and psychoeducation programmes, ${ }^{5}$ targeting 10- to 17 -year-old children and their families. Community-level non-psychiatrist workers have been trained to engage, interact with and deliver the intervention to the families, making sure that the children and women in the family are not ignored (as might otherwise happen in traditional, patriarchal nonWestern societies). Currently the model is undergoing pre-pilot initial acceptability and feasibility testing and iteration in rural locations in Kenya and India. Eventually, after further fine-tuning of the package and its delivery, it is hoped that the intervention will be able to enhance the resilience of the youth and families living in community, with a knock-on effect on mental health of the community.

Life skills are more to do with an individual, whereas family resilience approaches demand effort from all members. Integrating the individual (life skills) and family (resilience) models involves utilising the complementarity roles as individuals and also as family members, who, in turn, serve as a bridge between the individual and community-related resilience factors, especially in a non-Westernized LMIC setup. Both life skills and family resilience thus focus on dealing the challenges in life by identifying, utilising and enhancing the skills/strengths and reducing the risks/weakness through cognitive, emotional and social approaches. Our current approach proposes a five-session telephonic/video dialogue with families, involving story-telling; for example, where a scenario of a lowermiddle-class working family suddenly facing the COVD-19 crisis is used to guide the discussion on resilience. This is combined with elements of the LSE geared toward the children in the family. The five sessions are themed as rapport building and setting the agenda, belief systems, organisational processes, communication processes and closure with feedback.

The preliminary consultations on the model with young people, teachers and communities are promising in terms of the interest shown in the settings of both the countries, although our original plans for intensive consultations in faith communities and schools have had to be relegated (because of lockdown and other restrictions) to more in-depth and individual remote testing, but still with a focus on family and youth. The COVID-19 pandemic has forced us to consider how to make research efforts resilient and responsive to the needs of young people, to turn the worst of times into one of new opportunities, and realise that fundamental structural inequalities will also translate to greater vulnerability to natural disasters and crises like the pandemic. Our existing armoury of interventions may indeed have been engineered for the more privileged in higher-income countries, rather than the majority of young people around the world, who live in precarious conditions. As a research and practice community, we must join hands to collaborate and share knowledge and resources, and most importantly, foster an indomitable spirit in the face of adversity, which, interestingly, are the hallmarks of resilience.

Debasish Basu, Department of Psychiatry, Postgraduate Institute of Medical Education and Research, India; Sugandha Nagpal, Jindal School of International Affairs, O.P. Jindal Global University, India; Renjith R. Pillai, Department of Psychiatry, Postgraduate Institute of Medical Education and Research, India; Victoria Mutiso, Africa Mental Health Research and Training Foundation, Kenya; David Ndetei $\mathbb{D}$, Africa Mental Health Research and Training Foundation, Kenya; Department of Psychiatry, University of Nairobi, Kenya; Kamaldeep Bhui (iD, Department of Psychiatry, University of Oxford, UK; and Nuffield Department of Primary Care Health Sciences, University of Oxford, UK

Correspondence: Kamaldeep Bhui. Email: kam.bhui@psych.ox.ac.uk

First received 22 Jan 2021, final revision 5 Jul 2021, accepted 06 Jul 2021

\section{Acknowledgements}

This research described is funded by QMUL Global Challenges Research Fund, with UK, Kenyan and Indian partners working with the Global Policy Institute, and delivered in association with the UK and Kenyan World Psychiatric Association Collaborating Centres.

\section{Author contributions}

D.B. and K.B. drafted the editorial and received comments from all authors who commented on consecutive drafts and contributed to the work. K.B. is the principle investigator for the project. 


\section{Funding}

The editorial received no specific funding, but the project described was funded by QMUL's GCRF large grants to K.B.

\section{Declaration of interest}

K.B. is Editor-in-Chief of the British Journal of Psychiatry and did not take part in the review or decision-making process of this paper.

\section{References}

1 Fritz J, de Graaff AM, Caisley H, van Harmelen A-L, Wilkinson PO. A systematic review of amenable resilience factors that moderate and/or mediate the relationship between childhood adversity and mental health in young people. Front Psychiatry 2018; 9: 230.

2 Walsh F. Loss and resilience in the time of COVID-19: meaning making, hope, and transcendence. Fam Process 2020; 59(3): 898-911.

3 Pedersen GA, Smallegange $\mathrm{E}$, Coetzee A, Hartog K, Turner J, Jordans MJD, et al. A systematic review of the evidence for family and parenting interventions in low- and middle-income countries: child and youth mental health outcomes. Child Fam Stud 2019; 28: 2036-55.

4 Basu D, Nagpal S, Mutiso V, Ndetei DM, Lauwrens Z, Hadfield K, et al. Enhancing resilience and mental health of children and adolescents by integrated school- and family-based approaches, with a special focus on developing countries: a narrative review and call for action. World SOC Psychiatry 2020; 2: 7-19.

5 World Health Organization Division of Mental Health. Life Skills Education for Children and Adolescents in Schools (WHO/MNH/PSF/93.7A Rev. 2). World Health Organization, 1994 (https://apps.who.int/iris/handle/10665/63552). 